\title{
Uso de GeoGebraPrim para conjeturar y justificar en primaria ${ }^{1}$
}

\author{
Using GeoGebraPrim to guess and justify primary
}

\section{Usando GeoGebraPrim de adivinhar e justificar primárioa}

Recibido: mayo de 2013

Aceptado: agosto de 2013
Jenny Andrea Escobar ${ }^{2}$

Fredy Alejandro Barbosa ${ }^{3}$

Dra. Leonor Camargo Uribe 4

\section{Resumen}

Proponemos un taller para ilustrar cómo diseñar una secuencia de actividades que conllevan a la demostración de un teorema con niños de cuarto de primaria, con el fin que estudiantes en formación y profesores en ejercicio tengan un acercamiento experimental de cómo se utiliza un programa de geometría dinámica en particular GeoGebraPrim en prácticas de justificar y conjeturar en geometría plana a edades tempranas.

Palabras clave: Actividad demostrativa; primaria; secuencia de actividades; conjeturar; justificar; aula; recursos didácticos; recursos informáticos.

\begin{abstract}
We propose a workshop to illustrate how to design a sequence of activities that lead to the proof of a theorem with children from fourth grade, so that students in training and practicing teachers with an experimental approach of how to use a dynamic geometry program GeoGebraPrim particular to justify practices and conjecture in plane geometry at early ages.
\end{abstract}

Key words: Activity demonstrative; primary, sequence of activities, conjecture, justify; classroom teaching resources, resources.

\section{Resumo}

Propomos um workshop para ilustrar como criar uma seqüência de atividades que levam à demonstração de um teorema com crianças de quarta série, para que os alunos em formação e praticando professores com uma abordagem experimental de como usar um programa de geometria dinâmica GeoGebraPrim especial para justificar práticas e conjecturas em geometria plana em idades precoces.

Palavras chave: Atividade demonstrativo; primário, seqüência de atividades, conjectura, justificar; recursos didáticos em sala de aula, os recursos.

\footnotetext{
1 Artículo de Investigación.

2 Universidad Pedagógica Nacional. Bogotá, Colombia. Contacto: mdma_jescobar164@pedagogica.edu.co

3 Universidad Pedagógica Nacional. Bogotá, Colombia. Contacto: mdma_fbarbosa172@pedagogica.edu.co

4 Universidad Pedagógica Nacional. Bogotá, Colombia. Contacto: lcarmargo@pedagogica.edu.co, Profesora Universidad Pedagógica Nacional
} 


\section{Presentación}

Los estándares básicos de competencias en matemáticas sugieren que los estudiantes de educación básica primaria y secundaria tengan la posibilidad de construir y descomponer figuras, a partir de ciertas condiciones dadas, y conjeturar y verificar los resultados de aplicar transformaciones a figuras (MEN, 2006). En los lineamientos curriculares en matemáticas se promueve el reconocimiento de propiedades geométricas, relaciones e invariantes, a partir de la observación de regularidades y el establecimiento de conjeturas y generalizaciones, como aspectos importantes del hacer matemático escolar (MEN, 1998).

En busca de una alternativa para atender las directrices de los estándares y lineamientos, exploramos el uso del programa gratuito de geometría dinámica GeoGebraPrim el cual ha sido diseñado para estudiantes de primaria, el cual provee recursos apropiados a la edad de los niños para la investigación de propiedades geométricas. Tal exploración se hizo en el marco de una innovación educativa que apunta a crear un ambiente de aprendizaje que impulse procesos de conjeturación y justificación realizados por estudiantes de cuarto de primaria. Acompañamos el uso del programa de geometría dinámica con una secuencia de problemas que apunta al estudio de algunas propiedades de triángulos y cuadriláteros.

En el presente taller queremos proponer a educadores en formación y en ejercicio la secuencia de problemas, para desarrollarla en las sesiones conjuntas, e ir analizando cómo se puede orientar un proceso con estudiantes de primaria que los involucre en procesos de conjeturación y justificación.

\section{Marco teórico}

Tomando como punto de partida las ideas del grupo de investigación Aprendizaje y Enseñanza de la Geometría, Æ G, de la Universidad Pedagógica Nacional concebimos la actividad demostrativa para niños de cuarto de primaria como una práctica asociada a la resolución de problemas, que involucra dos procesos: la conjeturación y la justificación. Éstos se relacionan porque se busca justificar aquello que se conjetura. La conjeturación incluye detectar invariantes mediante la exploración, verificarlos y expresarlos como solución a un problema; el resultado, aun cuando no se exprese como una condicional, es tomado como una conjetura. La justificación busca la producción de un argumento que valide dicha conjetura, en un sistema de conocimiento compartido en la clase.

De acuerdo con Stylianides (2007) vemos la justificación como una demostración si ésta es un argumento matemático, una secuencia conectada de afirmaciones a favor de un enunciado y si las afirmaciones provienen de un sistema de conocimiento en el que los estudiantes:

- Usan hechos geométricos que son aceptados por la comunidad de la clase; esto quiere decir que los estudiantes los conocen, los aceptan y recurren a ellos cuando los necesitan.

- Usan formas de razonamiento que son válidas, conocidas y al alcance de la comunidad de la clase. En nuestra trayectoria, procuramos que los niños construyan enunciados condicionales en los que usen los hechos geométricos en situaciones específicas y eventualmente se refieran a los hechos geométricos como garantía de lo que afirman, desestimando el recurso a la ostensión.

- Usan formas comunicativas, es decir, notación, lenguaje simbólico, y expresiones al alcance de la comunidad de la clase. En nuestro caso, intentamos que los niños hagan uso del lenguaje y la notación de objetos de la geometría plana como segmentos, circunferencia, congruencia, bisecar, etc.

Una estrategia que hemos visto útil para lograr que los niños se involucren en la actividad demostrativa es proponer un conjunto de problemas tal que los hechos geométricos que se descubren y verifican 
en los primeros problemas, sirven de garantía para justificar los siguientes. Es en los últimos problemas en donde se lleva a cabo la actividad demostrativa propiamente dicha. Denominamos al conjunto de enunciados, resultado de la resolución de los problemas, como un sistema teórico local, compuesto por definiciones, postulados y teoremas que se van articulando unos con otros y de los cuales pueden valerse los niños para justificar enunciados. Gracias a un ambiente participativo y a la autonomía que promueve el profesor, los estudiantes se animan a expresar el resultado de sus exploraciones y a defender los resultados obtenidos, en el lenguaje y las formas de razonamiento aceptadas, y con sustento en los hechos geométricos establecidos en la clase.

\section{Descripción de la actividad}

El taller se realizará proponiendo a los asistentes seis problemas que deben resolverse con el apoyo del programa GeoGebraPrim. A medida que se van resolviendo, se irán identificando los hechos geométricos que conforman el sistema teórico local y cómo se articulan principalmente para justificar la conjetura que se obtiene en el problema seis. En la Tabla 1 se presentan las principales definiciones y hechos geométricos que surgen al trabajar con cada problema. En el Cuadro 1 se presenta un esquema de relaciones que esperamos sea discutido con los asistentes.

Tabla 1: Enunciados del Sistema teórico local

\begin{tabular}{|c|c|c|}
\hline Problema & Definiciones & Hechos geométricos \\
\hline 1 & $\begin{array}{l}\text { Radio } \\
\text { Congruencia }\end{array}$ & $\begin{array}{l}\text { HG 1: Si dos radios pertenecen a una misma circunferencia } \\
\text { son congruentes. }\end{array}$ \\
\hline 2 & Triángulo & $\begin{array}{l}\text { HG 2: si dos circunferencias son congruentes, sus radios son } \\
\text { congruentes. }\end{array}$ \\
\hline 3 & $\begin{array}{l}\text { Punto medio } \\
\text { Bisecar }\end{array}$ & \\
\hline 4 & $\begin{array}{l}\text { Diámetro } \\
\text { Rectángulo }\end{array}$ & $\begin{array}{l}\text { HG 3: todos los diámetros de una circunferencia son } \\
\text { congruentes. } \\
\text { HG4: si un cuadrilátero tiene diagonales congruentes y se } \\
\text { bisecan entonces es un rectángulo. }\end{array}$ \\
\hline 5 & $\begin{array}{l}\text { Triángulo } \\
\text { rectángulo }\end{array}$ & $\begin{array}{l}\text { HG 5: si el punto medio de un lado de un triángulo equidista } \\
\text { de los tres vértices entonces el triángulo es rectángulo. }\end{array}$ \\
\hline 6 & & $\begin{array}{l}\text { HG 6: si un triángulo está inscrito en una semicircunferencia } \\
\text { es rectángulo. }\end{array}$ \\
\hline
\end{tabular}

Fuente: Elaboración propia 
Cuadro 1:Relaciones entre los hechos geométricos de la secuencia de problemas

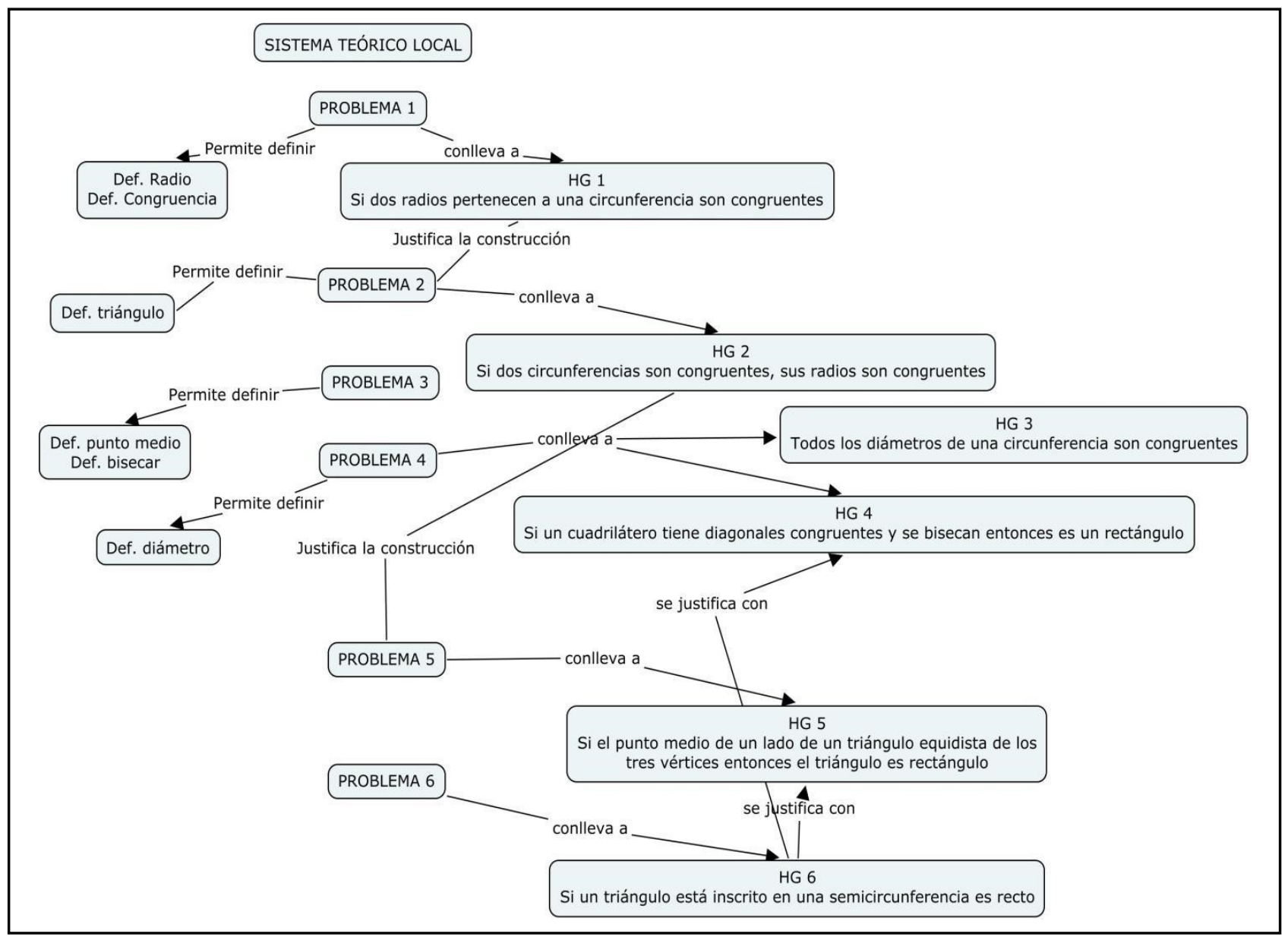

Fuente: Elaboración propia

\section{Reflexiones}

Se espera que el taller se discutan asuntos como el potencial que tiene el programa GeoGebraPrim para favorecer el trabajo en geometría de los estudiantes de primaria, la posibilidad de introducir a estudiantes de primaria en la actividad demostrativa y la posibilidad de que estudiantes tan pequeños construyan demostraciones.

\section{Referencias}

MEN. (1998). Lineamientos Curriculares para el área de matemáticas. Ministerio de Educación Nacional: Bogotá.
MEN. (2006). Estándares Básicos de Competencias en Lenguaje, Matemáticas, Ciencias y Ciudadanas Guía sobre lo que los estudiantes deben saber y saber hacer con lo que aprenden. Ministerio de Educación Nacional: Bogotá.

Stylianides, A.J. (2007). Proof and proving in school mathematics. Journal for Research in Mathematics Education, 38, 289-321. 